\title{
Special Issue: Advances in Architectures and Theories for Computer Vision
}

\author{
Yair Weiss $^{1} \cdot$ Vittorio Ferrari $^{2} \cdot$ Cristian Sminchisescu $^{2} \cdot$ Martial Hebert $^{3}$
}

Published online: 17 February 2020

(c) Springer Science+Business Media, LLC, part of Springer Nature 2020

Computer Vision has been revolutionized by deep learning, and it is not surprising that the overwhelming majority of our selected papers make extensive use of neural networks. This special issue focuses on advances in architectures and theories for computer vision. Often the contribution is a new architecture that is better suited to a particular vision task (e.g. Law and Deng 2019, Veit and Belongie 2019, Esteves et al. 2019) or the definition of a new visual task that can be tackled with this powerful tool (Harwath et al. 2019, Pumarola et al. 2019). The flow of ideas between Computer Vision and Deep Learning continues to be bidirectional, and one of the selected papers defines a new learning procedure that may prove to be useful beyond computer vision (Wu and He 2019). Over the last decade, the field of visual recognition has made the most use of machine learning methods, while geometry and reconstruction have mostly been less data-driven. In recent years we are witnessing a resurgence of learning based methods in geometry and reconstruction and three of our selected papers apply deep learning for pose estimation and modeling of 3D geometry (Sundermeyer et al. 2019, Zhou et al. 2019, Li et al. 2019). While the field of computer vision is dominated by empirical observations in recent years, three of our selected papers are more conceptual. They either rigorously examine the complexity of a classic algorithm (Chin et al. 2019) or present novel formulations of classic problems when applied to new sensors (Ma et al. 2019, Gehrig et al. 2019).

The idea for a special issue about architectures and theories for computer vision came from the ECCV conference held in 2018. Out of the 59 papers presented at the conference, 12 were selected for this special issue after being

Yair Weiss

yweiss@cs.huji.ac.il

1 Hebrew University of Jerusalem, Jerusalem, Israel

2 Google Research, Zurich, Switzerland

3 Carnegie Mellon University, Pittsburgh, PA, USA vetted against journal standards and going through the rigorous peer review process. Three of the papers were selected by the ECCV 2018 Best Paper Committee: Thomas Brox, Andrew Fitzgibbon, Alexei Efros, David Jacobs and Xiaoou Tang. The remaining nine papers were selected by the program chairs of the conference. Selected papers underwent an additional round of rigorous reviewing and revisions. Taken together, the picture that emerges from these 12 selected journal papers is that of a community that continues to adapt to new tools, but also contributes to the development of the tools in a way that is informed by the specific challenges of computer vision.

\section{References}

Chin, T. J., Cai, Z., \& Neumann, F. (2019). Robust fitting in computer vision: Easy or hard? International Journal of Computer Vision. https://doi.org/10.1007/s11263-019-01207-y.

Esteves, C., Allen-Blanchette, C., Makadia, A., \& Daniilidis, K. (2019). Learning SO(3) equivariant representations with spherical CNNs. International Journal of Computer Vision. https://doi. org/10.1007/s11263-019-01220-1.

Gehrig, D., Rebecq, H., Gallego, G., \& Scaramuzza, D. (2019). EKLT: Asynchronous photometric feature tracking using events and frames. International Journal of Computer Vision. https://doi. org/10.1007/s11263-019-01209-w.

Harwath, D., Recasens, A., Surís, D., Torralba, A., \& Glass, J. (2019). Jointly discovering visual objects and spoken words from raw sensory input. International Journal of Computer Vision. https:// doi.org/10.1007/s11263-019-01205-0.

Law, H., \& Deng, J. (2019). CornerNet: Detecting objects as paired keypoints. International Journal of Computer Vision. https://doi. org/10.1007/s11263-019-01204-1.

Li, Y., Wang, G., Ji, X., Xiang, Y., \& Fox, D. (2019). DeepIM: Deep iterative matching for $6 \mathrm{D}$ pose estimation. International Journal of Computer Vision. https://doi.org/10.1007/s11263-019-01250-9.

Ma, S., Smith, B. M., \& Gupta, M. (2019). Differential scene flow from light field gradients. International Journal of Computer Vision. https://doi.org/10.1007/s11263-019-01230-z. 
Pumarola, A., Agudo, A., Martinez, A. M., Sanfeliu, A., \& MorenoNoguer, F. (2019). GANimation: One-shot anatomically consistent facial animation. International Journal of Computer Vision. https://doi.org/10.1007/s11263-019-01210-3.

Sundermeyer, M., Marton, Z. C., Durner, M., \& Triebel, R. (2019). Augmented autoencoders: Implicit 3D orientation learning for 6D object detection. International Journal of Computer Vision. https ://doi.org/10.1007/s11263-019-01243-8.

Veit, A., \& Belongie, S. (2019). Convolutional networks with adaptive inference graphs. International Journal of Computer Vision. https ://doi.org/10.1007/s11263-019-01190-4.
Wu, Y., \& He, K. (2019). Group normalization. International Journal of Computer Vision. https://doi.org/10.1007/s11263-019-01198-w.

Zhou, H., Ummenhofer, B., \& Brox, T. (2019). DeepTAM: Deep tracking and mapping with convolutional neural networks. International Journal of Computer Vision. https://doi.org/10.1007/s11263-01901221-0. 